\title{
Comparative study of hematological parameters in newly diagnosed tuberculosis patient's pre-att \& after intensive phase of ATT
}

\author{
Manjunath M. R., , Sheetal2,", Abdul Rub Patwegar ${ }^{3}$ \\ ${ }^{\mathbf{1}}$ Associate Professor, ${ }^{2}$ Postgraduate Student, ${ }^{3}$ Medical Student, ${ }^{1,2}$ Dept. of Pathology, ${ }^{1-3}$ Mandya Institute of Medical Science \\ Mandya, Karnataka, India
}

*Corresponding Author:

Email: sheetusudha@gmail.com

\begin{abstract}
Introduction: Tuberculosis is a highly prevalent chronic infectious disease. TB exerts various hematologic effects \& hematological abnormalities. Anti-Tuberculosis treatment has its own spectrum of effects on TB patients. This study aimed to study \& compare various hematological parameters in newly diagnosed TB patients before ATT \& to evaluate after the intensive phase ATT in same patient.

Materials and Methods: A total of 40 newly diagnosed cases of TB were randomly selected. Under aseptic precautions, $2.5 \mathrm{ml}$ of blood collected from patients in EDTA vacutainers \& the following hematological parameters were analysed - HB\%, TLC, DC, PC, MCV, MCHC, PCV and ESR. The same patients were put on 2 months of intensive phase of ATT. After completion of ATT, the hematological parameters of each patient were analysed. PreATT and after ATT the parameters were compared and analyzed with suitable statistics.

Results: There was significant post treatment improvements in Hb\%(Pre ATT-10g/dl \& post ATT 11.8g/dl), RBC count (Pre ATT-4.8 millions/cumm \& Post ATT-4.85 millions/cumm), PCV (Pre ATT-38\% \& Post ATT-40\%), Total leucocyte count (Pre ATT-10,295cells/cumm \& post ATT- 9,500 cells /cumm), Neutrophils (Pre-ATT-73\% \& post ATT-63\%) and Lymphocytes (PRE-ATT - $20 \%$ \& post ATT-30\%) and ESR (PreATT $79 \mathrm{~mm} / \mathrm{hr} \&$ post ATT $54 \mathrm{~mm} / \mathrm{hr}$ ). The present study showed that, there was a significant statistical difference in $\mathrm{HB} \%$, TLC, DC, Platelets \& ESR values.

Conclusion: Hematological parameters can be a guide to assess the improvement of TB patients after ATT which indirectly speaks about the improvement in patients.
\end{abstract}

Keywords: TB, ATT.

\section{Introduction}

Tuberculosis is a highly prevalent chronic infectious disease caused by Mycobacterium tuberculosis. Tuberculosis infection remains at an epidemic level affecting one third of world population. ${ }^{1}$ Globally, there are 8 million new cases and 3 million deaths annually due to pulmonary tuberculosis $[\mathrm{PTB}] .^{2}$ Tuberculosis is a major public health problem in India affecting about one third of the population. ${ }^{3}$

The diagnosis of PTB mainly relies on detection of acid-fast bacilli in sputum smears and cultures. Active PTB may produce signs and symptoms that include hematological abnormalities, hypernatremia and psychological disorders. Hematological parameters are altered during infections. Complete blood picture is the routine investigation done for every patient irrespective of the type of infection that provides much needed information for making decision of treatment. In tuberculosis, various hematological derangements are seen including low hemoglobin, decreased lymphocyte count with its subsets, neutrophilia, monocytosis, monocytopenia, thrombocytopenia and thrombocytosis in few cases. ${ }^{2}$ Reversible peripheral blood abnormalities are commonly associated with pulmonary tuberculosis. Insight into the relationship between hematological abnormalities and mycobacterial infection has come from an understanding of the immunology of mycobacterial infection. ${ }^{4}$

Though tuberculosis is a common condition, the atypical and varied spectrum of clinical presentation of tuberculosis poses a diagnostic and therapeutic challenge to the physicians. Also, little is known about the prevalence of these hematological abnormalities and the effect of Anti-Tuberculosis Treatment [ATT] on the various hematological parameters in the Indian subcontinent. This study was undertaken to analyze the hematological parameters and their variations in PTB patients with sputum smear positive patients before initiation of ATT and to evaluate their diagnostic significance with initial phase of ATT.

\section{Materials and Methods}

This study was done in the Department of Pathology, Mandya Institute of Medical Sciences and Teaching Hospital, Mandya, in duration of 3 months from July to September, 2017.

A total of 40 newly diagnosed cases of tuberculosis were randomly selected and subjected to detailed medical history, general physical and systemic examination with the consent taken from the patients. New cases of pulmonary tuberculosis with sputum smear positive, between the age group of 15-65 years were included in this study._Pulmonary tuberculosis patients 
who were already receiving treatment, tuberculosis patients with HIV, with any other complications like malignancy or other systemic disorders were excluded from the study.

Sample collection: A total of 40 new cases were included in this study. Under aseptic precautions, $2.5 \mathrm{ml}$ of venous blood was collected from all the patients in EDTA vacutainers. The blood thus collected was processed in 2 ways:

1. Automated cell count by using ABACUS hematology analyzer which utilizes laser based optical measurement technology to give 5-part differential results. ${ }^{8}$

2. Preparation of thin blood smears and the smears are stained by Leishman stain for physical analysis of blood cell morphology and to verify the values of cell counts given by the automated cell count analyzer. $^{9}$

Following hematological parameters were obtained with both ways of processing of the blood samples- hemoglobin concentration, total leucocytes count, differential leucocytes counts, absolute eosinophil count, platelet counts, MCV, MCHC, PCV and ESR.

Then the same patient was put on initial two months of intensive phase of ATT. After the completion of 2 months of ATT again the same process of blood collection and processing of blood samples was done on the same individuals. The two hematological parameters of each patient were compared and analyzed.

\section{Results}

The present study was done on 40 newly diagnosed cases of pulmonary tuberculosis patients. Out of them 10 (25\%) participants were aged 20 to 35 years, $18(45 \%)$ participants were aged 36 to 50 years and 12 (30\%) participants were aged 51 to 65 years. Among the study population, $29(72.50 \%)$ participants were male and remaining $11(27.50 \%)$ participants were female.

Table 1: Following are the averages of the hematological parameters of TB patients before the initiation of ATT and after the completion of intensive phase of ATT

\begin{tabular}{|l|c|c|c|}
\hline \multicolumn{1}{|c|}{ Parameter } & $\begin{array}{c}\text { Before ATT } \\
\text { Median (IQR) }\end{array}$ & $\begin{array}{c}\text { After ATT } \\
\text { Median (IQR) }\end{array}$ & $\begin{array}{c}\text { P value (Wilcoxon } \\
\text { signed Test) }\end{array}$ \\
\hline RBC millions/mm ${ }^{3}$ & $4.80(4.37$ to 5.20$)$ & $4.85(4.25$ to 5.30$)$ & 0.011 \\
\hline Haemoglobin (g/dl) & $10(8.40$ to 12.57$)$ & $11.80(10.80$ to 12.90$)$ & $<0.001$ \\
\hline PCV & $38(32$ to 44.75$)$ & $40(38$ to 46.25$)$ & 0.006 \\
\hline WBC & $10295(3800$ to 13000$)$ & $9500(4510$ to 11330$)$ & 0.306 \\
\hline Neutrophils & $73(40$ to 89$)$ & $63(60$ to 70$)$ & 0.475 \\
\hline Lymphocytes & $20(10$ to 59$)$ & $30(26$ to 34$)$ & 0.606 \\
\hline Monocytes & $1(1$ to 2$)$ & $3(2$ to 4$)$ & $<0.001$ \\
\hline Eosinophils & $1(0$ to 2$)$ & $2(0$ to 3$)$ & 0.002 \\
\hline Basophils & $0(0$ to 0$)$ & $0(0$ to 0$)$ & 1.000 \\
\hline ESR in mm/ hours & $79(70.25$ to 86$)$ & $54(43$ to 62$)$ & $<0.001$ \\
\hline
\end{tabular}

Among the study population, the median RBC before ATT was 4.80 (IQR 4.37 to 5.20) and after intensive phase of ATT was 4.85 (IQR 4.25 to 5.30). The difference was statistically significant (P Value 0.011).

The median haemoglobin before ATT was $10 \mathrm{~g} / \mathrm{dl}$ (IQR 8.40 to 12.57) and after intensive phase of ATT the median haemoglobin was $11.80 \mathrm{~g} / \mathrm{dl}$ (IQR 10.80 to 12.90). The difference was statistically significant $(P$ Value $<0.001$ ).

The median PCV was 38 (IQR 32 to 44.75) before ATT and it was 40 (IQR 38 to 46.25 ) after intensive phase of ATT. The difference was statistically significant (P Value 0.006).

The difference of values of WBC total counts in between before ATT and after intensive phase of ATT was statistically not significant (P Value 0.306).

The difference in the values of differential counts of neutrophils before ATT and after intensive phase of ATT was statistically not significant (P Value 0.475).

The difference in between the values of differential counts of lymphocytes before ATT and after intensive phase of ATT was statistically not significant (P Value 0.606).

Among the study population, the median monocytes was 1 (IQR 1 to 2) before ATT and it was 3 (IQR 2 to 4 ) after intensive phase of ATT. This difference in between before ATT and after ATT of monocytes differential counts were statistically significant (P Value $<0.001$ ).

Among the study population, the median eosinophils was 1 (IQR 0 to 2) before ATT and it was 2 (IQR 0 to 3 ) after intensive phase of ATT. This difference was statistically significant (P Value 0.002).

The difference in the differential counts of basophils before ATT and after ATT was statistically not significant (P Value 1.000).

Among the study population, the median ESR was $79 \mathrm{~mm} /$ hours (IQR70.25 to 86) before ATT and it was $54 \mathrm{~mm} /$ hours (IQR 43 to 62) after intensive phase of ATT. This difference in ESR values was statistically significant (P Value $<0.001)$. 


\section{Discussion}

Tuberculosis exerts varieties of hematologic effects. Hematological abnormality is a common finding among TB patients. Anti-Tuberculosis treatment has its own spectrum of effects on tuberculosis patients. The current study evaluated the hematological parameters of TB patients before initiation of intensive phase of ATT and after completion of intensive phase of ATT.

Effects on Red Blood Cell Count: In the current study, the average RBC counts before ATT is 4.80 millions/cumm, whereas the average RBC counts after ATT is 4.856 millions/cumm. There was statistical significant difference (P Value 0.011) in the RBC count. This correlates with the study conducted by Eyuel $\mathrm{K}$ et al in university of Gondar hospital, Ethiopia on 168 patients where average RBC count of TB patients before and after completion of intensive phase of ATT were $4.25 \mathrm{x}$ $10^{3}$ and $4.42 \times 10^{3}$ respectively. ${ }^{6}$

In this study most of the patients (23 out of 40 patients - i.e 52.5\%) had anemia before starting treatment. This correlated with study of Parasappa J Y et al on 100 PTB patients where anemia was seen in 74 patients before ATT. ${ }^{1}$ The study conducted by Sumaira I et al on 45 tuberculosis patients gives the conclusion that anemia is one of the commonest findings seen in TB patients and is considered to be responsible for poor prognosis. $^{3}$

In this study most common type of anemia being Normocytic Normochromic anemia [42.8\%] and second common anemia was Microcytic Hypochromic anemia
[33.3\%], rest having macrocytic normochromic anemia [23.8\%]. This correlated with the study conducted by Shidram K et al on 100 tuberculosis patients; the commonest type of anemia was Normocytic Normochromic anemia in $84 \%$ of their cases before ATT. ${ }^{5}$

In this study, after treatment, the hemoglobin levels improved significantly. Out of the 23 patients who had anemia, the hemoglobin levels of 14 patients improved significantly after ATT which also correlated with the study conducted by Shidram K et al with statistically meaningful results of pvalue $<0.001 .^{5}$

In our study there is a statistically significant difference (P Value $<0.001$ ) in hemoglobin concentration in females and males before and after treatment with intensive phase of ATT.

Table 2: Comparison of median hemoglobin before and after intensive phase of ATT between males and females $(\mathrm{N}=40)$

\begin{tabular}{|l|c|c|c|}
\hline \multirow{2}{*}{ Gender } & \multicolumn{2}{|c|}{ Hemoglobin (g/dl) } & Mann \\
\cline { 2 - 3 } & $\begin{array}{c}\text { Before ATT } \\
\text { Median } \\
\text { (IQR) }\end{array}$ & $\begin{array}{c}\text { After ATT } \\
\text { Median } \\
\text { (IQR) }\end{array}$ & $\begin{array}{c}\text { U test } \\
\text { (P value) }\end{array}$ \\
\hline Male & $\begin{array}{c}11.40(9.20 \\
\text { to } 12.70)\end{array}$ & $\begin{array}{c}12(11 \text { to } \\
13.25)\end{array}$ & 0.002 \\
\hline Female & $\begin{array}{c}8.20(7.80 \text { to } \\
10)\end{array}$ & $\begin{array}{c}10.80(10.60 \\
\text { to } 11)\end{array}$ & 0.028 \\
\hline
\end{tabular}

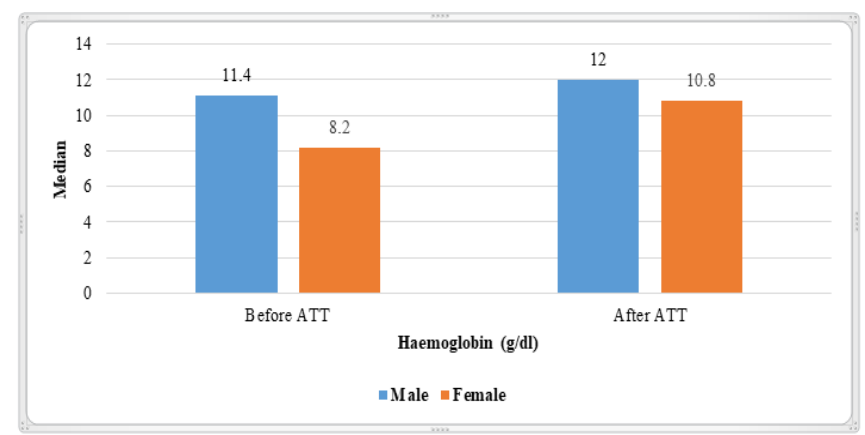

Fig. 1: Bar diagram comparison of median haemoglobin before and after invention between gender combination $(\mathbf{N}=\mathbf{4 0})$

Effects on Total Leucocyte Count: In this study, 14 [35\%] patients had leucocytosis [WBC count more than $12,000], 9[22.5 \%]$ patients had leucopenia [WBC count $<4,000]$ and 17 [42.5\%] patients had normal WBC count in normal range $[4,000-12,000]$ before initiation of ATT.

After intensive phase of ATT, 35 [87.5\%] patients had normal leucocyte count and 5[12.5\%] patients had leucocytosis. Out of the 14 patients who had leucocytosis before ATT, 9 patient's WBC count had significant improvements to normal levels after treatment with ATT.
This correlates with the study conducted by Shidram $\mathrm{K}$ et al, where most of the patients $(92 \%)$ had a total leucocyte count (TLC) of more than $11,000 / \mathrm{mm}^{3}$ before treatment, Normal TLC in 5\% and $<4,000 /$ cumm in $3 \%$ cases. After treatment there is strongly significant improvement in TLC $(\mathrm{p}<0.001) .{ }^{5} \mathrm{Al}$ Omar et al 2009, also observed that leucocytosis observed initially in the PTB patients in the study became normal both in male and female patients. ${ }^{10}$ 
Table 3: Descriptive analysis of WBC before and after ATT in the study population ( $N=40)$

\begin{tabular}{|l|c|c|}
\hline WBC Count & Before ATT & After ATT \\
\hline$<4000$ & $9(22.50 \%)$ & $0(0 \%)$ \\
\hline 4000 to 11000 & $17(42.50 \%)$ & $35(87.5 \%)$ \\
\hline$>11000$ & $14(35.00 \%)$ & $5(12.5 \%)$ \\
\hline
\end{tabular}

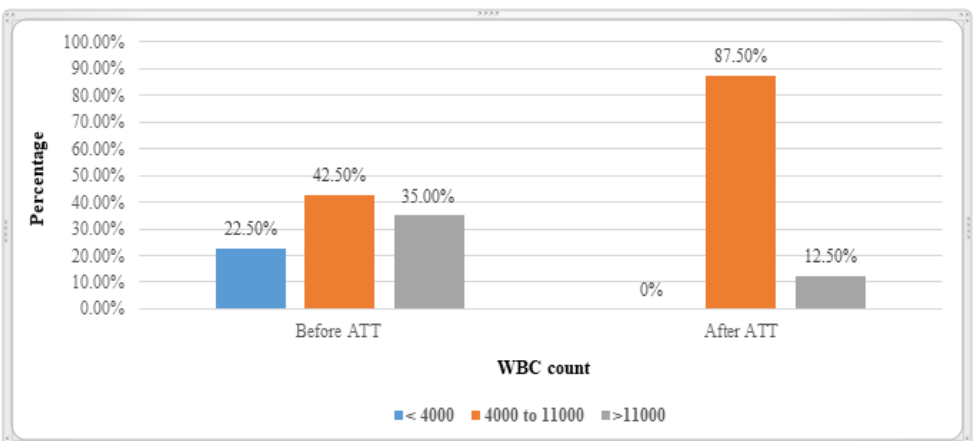

Fig. 2: Bar diagram of descriptive analysis of WBC after ATT and WBC before ATT (N=40)

Effect on Neutrophil: In this study, 17 [42.50\%] patients had neutrophilia [>75\%], 12 [30\%] patients had neutropenia $[<50 \%]$ and $11[10 \%]$ patients had normal neutrophil count [50-75\%] before initiation of ATT. After completion of intensive phase of ATT all of the patient's neutrophil counts came back to normal ranges.

Out of 17 patients who had neutrophilia before ATT, the neutrophil count of all the 17 patients was significantly reduced to normal limits. This correlates with the values of the study conducted by Shidram K et al, where in their study on 100 PTB patients, 35 patients had neutrophilia before ATT and after ATT, 33 patient's neutrophil count got reduced to normal limits. ${ }^{5}$
Table 4: Descriptive analysis of neutrophils before and after ATT in the study population ( $=40)$

\begin{tabular}{|l|c|c|}
\hline Neutrophils & Before ATT & After ATT \\
\hline$<50$ & $12(30.00 \%)$ & $0(0 \%)$ \\
\hline 50 to 75 & $11(27.50 \%)$ & $40(100 \%)$ \\
\hline$>75$ & $17(42.50 \%)$ & $0(0 \%)$ \\
\hline
\end{tabular}

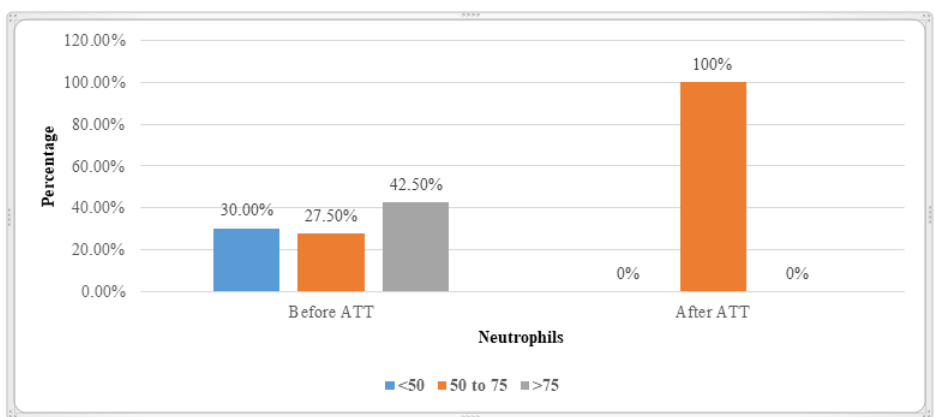

Fig. 3: Bar chart of descriptive analysis of neutrophils before and after ATT in the study population (N=40)

Effect on Lymphocyte Percentage: In this study, $16(40 \%)$ patients had lymphocytopenia $(<20 \%)$, normal count [20-40\%] in 12[30\%] cases and rest 12 [30\%]having lymphocytosis $(>50 \%)$.

Post treatment there is significant improvement in lymphocyte count. Out of 16 patients who had lymphocytopenia before ATT, 13 patients' lymphocyte count was reduced to normal after ATT and out of the
12 patients who had lymphocytosis before ATT, 11 patients' lymphocyte count was reduced to normal levels after ATT. This correlates with the study of Shidram K et al, where in their study on 100 PTB patients 49 had lymphocytopenia, normal count in 43 cases and rest having lymphocytosis. Post treatment there is significant improvement in lymphocyte count with a $p$ value of $0.05-0.1 .^{5}$ 
Table 5: Descriptive analysis of lymphocytes before and after ATT in the study population $(\mathrm{N}=40)$

\begin{tabular}{|c|c|c|}
\hline Lymphocytes & Before ATT & After ATT \\
\hline$<20$ & $16(40.00 \%)$ & $3(7.5 \%)$ \\
\hline 20 to 40 & $12(30.00 \%)$ & $36(90 \%)$ \\
\hline$>40$ & $12(30.00 \%)$ & $1(2.5 \%)$ \\
\hline
\end{tabular}

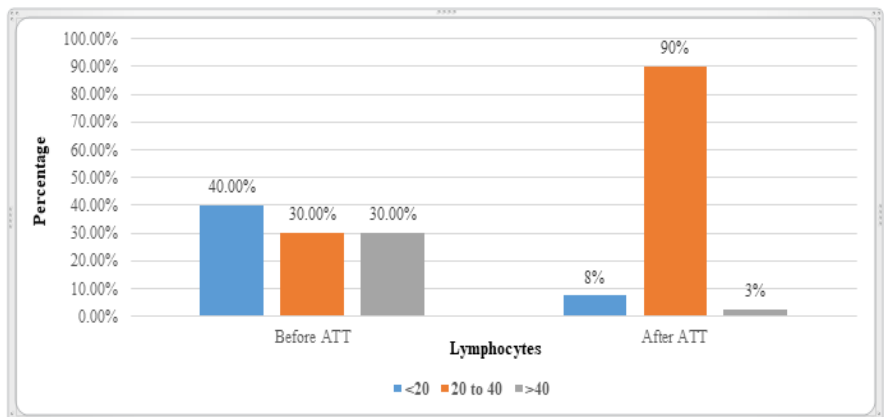

Fig. 4: Bar chart of descriptive analysis of lymphocytes before and after ATT in the study population (N=40)

Effect on Monocyte Percentage: In this study 28 (70\%) patients had monocytopenia before intensive phase of ATT and 12 (30\%) patients had normal monocyte counts before intensive phase of ATT. After intensive phase of ATT, all the 28 patients who had monocytopenia, the monocyte counts improved to a normal range. This correlated with Lombard et al where in there study monocytopenia was recorded in $50 \%$ of the study population. ${ }^{12}$
Table 6: Descriptive analysis of monocytes before and after ATT in the study population $(\mathrm{N}=40)$

\begin{tabular}{|c|c|c|}
\hline Monocytes & Before ATT & After ATT \\
\hline$<2$ & $28(70.00 \%)$ & $0(0 \%)$ \\
\hline 2 to 10 & $12(30.00 \%)$ & $40(100 \%)$ \\
\hline
\end{tabular}

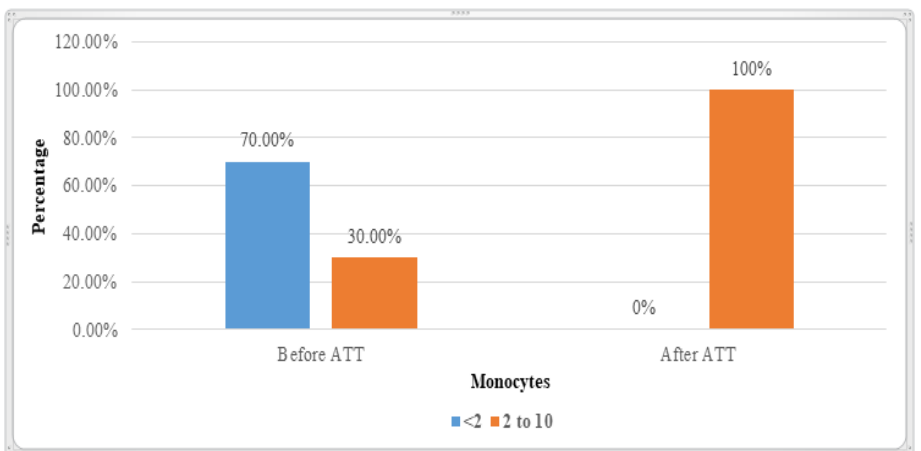

Fig. 5: Bar chart of descriptive analysis of monocytes before and after ATT in the study population (N=40)

Effect on Platelet Count: In this study a majority of patients [34 patients $(85 \%)]$ had normal level of platelet count and $6[15 \%]$ patients had thrombocytopenia. This correlates with the study of Maartens et al, 1990 which reports 24 out of 104 (23\%) cases of thrombocytopenia with Tuberculosis. ${ }^{11}$

Post treatment there is significant change in platelet count in patients with thrombocytopenia where out of the 6 patients who had thrombocytopenia before ATT, 5 patient's platelet count improved after ATT. This correlates with the study of Shidram K et al where out of 100 patients in their study, 33 patients who had thrombocytopenia before ATT, all 33 patients platelet counts was normalized post treatment with ATT. ${ }^{5}$

Table 7: Descriptive analysis of platelet count before and after ATT in the study population $(\mathrm{N}=40)$

\begin{tabular}{|l|c|c|}
\hline Platelet count & Before ATT & After ATT \\
\hline $\begin{array}{l}\text { Thrombocytopenia } \\
\text { [<1.5LAKHS/cumm }]\end{array}$ & $6(15.00 \%)$ & $1(2.5 \%)$ \\
\hline $\begin{array}{l}\text { Normal platelet count } \\
\text { [1.5-4LAKHS/cumm] }\end{array}$ & $34(85.00 \%)$ & $39(97.5 \%)$ \\
\hline
\end{tabular}




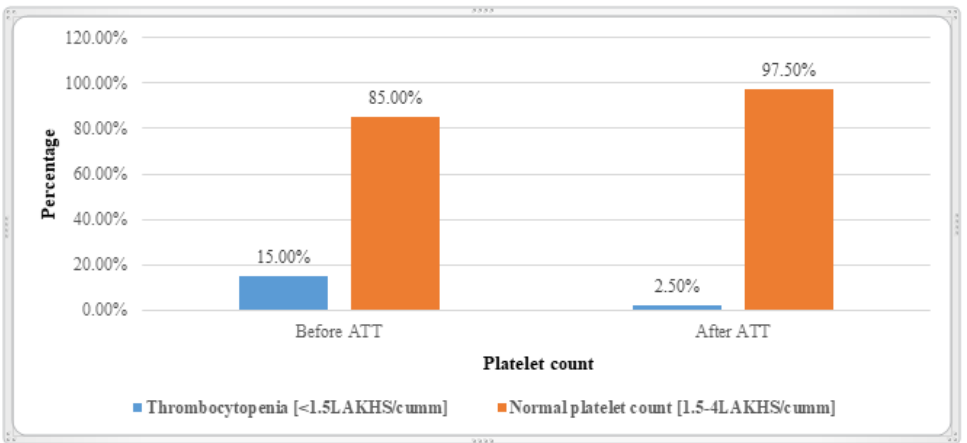

Fig. 5: Bar chart of descriptive analysis of platelet count before and after ATT in the study population (N=40)

Effects on Erythrocyte Sedimentation Rate (Esr): In this study, all the cases had increased ESR before treatment which correlates with Parasappa J Y, where all 100 patients in their study showed a significant increase in ESR before ATT. ${ }^{1}$

Post treatment there is significant reduction in ESR values which correlates with study of Shidram $\mathrm{K}$ et al where in their study all 100 patients of PTB who were included showed an increase in ESR pre-ATT whereas post treatment there was a significant change in ESR with a p-value of 0.005 .5 Also Morris et al study showed increased ESR in $80 \%$ patients with pulmonary tuberculosis. ${ }^{10}$ The ESR value at post treatment were extremely significant $(\mathrm{p}<0.0001)$ with treatment denotes gradual decrease in TB infection and good response to treatment. ${ }^{5,10}$

Table 8: Descriptive analysis of ESR values before and after in the study population $(\mathrm{N}=40)$

\begin{tabular}{|c|c|c|}
\hline Platelet count & Before ATT & After ATT \\
\hline High ESR & $40(100 \%)$ & $0(0 \%)$ \\
\hline Low ESR & $0(0 \%)$ & $40(100 \%)$ \\
\hline
\end{tabular}

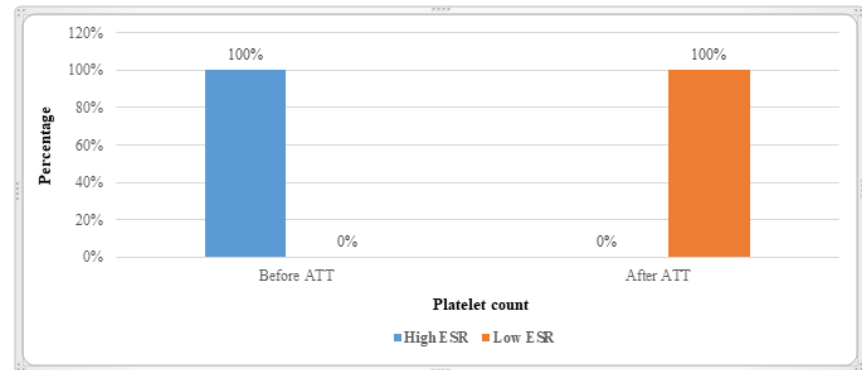

Fig. 6: Bar chart of descriptive analysis of ESR values before and after in the study population $(\mathrm{N}=40)$

\section{Conclusion}

In this study there are significant post treatment changes in $\mathrm{Hb} \%$, Total leucocyte count, percentage of Neutrophils, Lymphocytes, monocytes, Platelet counts and ESR. Hence, Anti-Tuberculosis Drug Treatment [ATT] is very effective in improving hematological parameters which indirectly speaks about the improvement in immune response. But as this study involves lesser patients, it has to be done with more number of patients to evaluate further.

\section{Conflict of Interest: None}

\section{References}

1. Parasappa JY, Toolhally U, Sadula G H. Hematological Profile in Pulmonary Tuberculosis. Int J Health Rehabil Sci 2013;2(1):50-5.

2. Hager A F, Nizar R M A. Abnormalities of hematological parameters in newly diagnosed Pulmonary tuberculosis patients in Kirkuk city. J Babylon Univ/Pure Appl Sci 2012;20(5)
3. Sumaira I, Umbreen A, Muhammad A K. Hematological parameters altered in tuberculosis. PakJ Physio. 2015;11(1)

4. Atomsa D, Abebe G, Sewunet T. Immunological Markers and Hematological Parameters among Newly Diagnosed Tuberculosis Patients at Jimma University Specialized Hospital. Ethiop J Health Sci 2014;24(4):311-18.

5. Shidram K, Ramesh B, Bhaktavatchalam N. Study of hematological profile before during after completion of dots therapy in pulmonary tuberculosis. J Evid Based Med Healthc 2014;1(8):962-68.

6. Mohammed A, Saeed M. Some Hematological Parameters among Patients with Pulmonary Tuberculosis. J App Med Sci 2016;4(1B):99-111

7. Eyuel k, Bamlaku E, Aschalew G, Baye G. Effects of anti-tuberculosis drugs on hematological profiles of tuberculosis patients attending at university of Gondar Hospital, Northwest Ethiopia. BMC Hematol 2016

8. Graham MD. The Coulter Principle: Foundation of an Industry. J Lab Automation 2003;8(6):72-81. 7.

9. Wittekind DH, Kretschmer V, Sohmer I. Azure B- eosin Y stain as a standard Romanowsky - stain. Br J Haematol 1982;5:391-93 
10. Morris CDW, Bird AR, Nell H. The hematological and biochemical changes in severe pulmonary tuberculosis. Quint J Med 1989;272:1151-59.

11. Maartens. G, A. Paul, R. Willcox, S. Benater, 1990, Miliary tuberculosis. Rapid diagnosis, hematological abnormalities and outcome in 109 treated adults. $J$ Clin Invest 1990;89(3):291-96. PMID: 2393033.

12. Lombard, E.H. E.P.G. Mansvelt, 1993. Hematological changes associated with miliary tuberculosis of bone marrow. Tuberc Lung Dis 74(2):131-35. PMID; 8324206
How to cite the article: Manjunath $M$. R, Sheetal, Patwegar A.R. Comparative study of hematological parameters in newly diagnosed tuberculosis patient's preatt $\&$ after intensive phase of ATT. Arch Cytol Histopathol Res 2018;3(4):185-191. 